\title{
Luteolin sensitizes human liver cancer cells to TRAIL-induced apoptosis via autophagy and JNK-mediated death receptor 5 upregulation
}

\author{
UDDIN MD. NAZIM and SANG-YOUEL PARK \\ Biosafety Research Institute, College of Veterinary Medicine, \\ Chonbuk National University, Iksan, Jeonbuk 54596, Republic of Korea \\ Received February 26, 2018; Accepted September 19, 2018
}

DOI: $10.3892 /$ ijo.2018.4633

\begin{abstract}
The tumor necrosis factor-related apoptosis-inducing ligand (TRAIL) is a dynamic cytokine that initiates the apoptosis of cancer cells, but exhibits little or no toxicity in normal cells. Luteolin is a flavonoid compound frequently used in the treatment of cancer. In the current study, we demonstrate that treatment with luteolin and TRAIL exerts a synergistic effect and the mechanisms on TRAIL-resistant Huh7 cells. The results demonstrated that luteolin induced an autophagic flux in human liver cancer cells. The attenuation of the autophagic flux by applying the specific inhibitor of autophagy, chloroquine, significantly suppressed DR5 expression. Treatment with genetically modified autophagy-related 5 siRNA abrogated the luteolin-mediated sensitizing effect of TRAIL. Furthermore, pre-treatment with the c-Jun N-terminal kinase (JNK) inhibitor, SP600125, significantly attenuated the luteolin-induced upregulation of DR5 expression, thereby suggesting that JNK activation promotes DR5 expression. Our findings also revealed that Akt phosphorylation was required for TRAIL sensitization. On the whole, the findings of this study indicated that luteolin effectively enhanced TRAIL-initiated apoptosis, and that these effects were likely to be mediated by autophagy and JNK-mediated DR5 expression.
\end{abstract}

\section{Introduction}

Hepatocellular carcinoma (HCC) is the third leading cause of cancer-related mortality worldwide $(1,2)$. Remedial therapies for HCC, such as surgery, radiofrequency ablation, liver transplantation and chemotherapy, remain limited (3).

Correspondence to: Dr Sang-Youel Park, Biosafety Research Institute, College of Veterinary Medicine, Chonbuk National University, Gobong-ro 79, Iksan, Jeonbuk 54596, Republic of Korea E-mail: sypark@chonbuk.ac.kr

Key words: luteolin, autophagic flux, tumor necrosis factor-related apoptosis-inducing ligand, cancer, c-Jun N-terminal kinase, death receptor-5
Nevertheless, surgery may have adverse effects and is not suitable for patients with advanced disease $(4,5)$. Advanced tumors are not usually resistant to single-sequence therapy; however, combination chemotherapy can prolong the 5-year survival rate and reduce recurrence rates.

Tumor necrosis factor (TNF)-related apoptosis-inducing ligand (TRAIL) is a type II transmembrane protein and a member of the TNF superfamily. TRAIL selectively initiates the apoptosis of cancer cells without apparent toxic side-effects on normal cells, thus making it a promising chemotherapeutic agent for the treatment of various types of cancer (6-9). However, recent findings have revealed that some cancer cells, including HCC cells, can develop resistance to TRAIL-mediated apoptosis (10). TRAIL crosslinks to its receptors, namely death receptor (DR)4 and DR5, and triggers their trimerization and intracellular adaptor death domain clustering to generate a death-inducing signaling complex $(11,12)$. This complex then recruits the Fas-associated death domain accessory molecule and subsequently incites caspase- 8 . In turn, caspase-8 incites downstream 'effector' caspases, such as caspase-3 (13).

Luteolin (chemical name, 3',4',5,7-tetrahydroxyflavone) is a widely used flavonoid compound produced in numerous types of plants, such as vegetables, fruits and medicinal herbs. Previous studies have revealed that luteolin exhibits diverse biological properties, such as antioxidant (14), anti-inflammatory (15) and anti-proliferative effects (16). The anticancer effects of luteolin have been demonstrated in numerous cancer cells, such as prostate, pancreatic, colorectal, breast and ovarian cancer cells (17-20). It has been demonstrated that luteolin, as a flavonoid, can cross the blood brain barrier $(21,22)$. However, the regulatory mechanisms underlying the effects of luteolin in HCC cells remain unclear (23).

Autophagy is a catabolic degradation mechanism that results in the autophagosomal-lysosomal degeneration of cytosolic proteins and other cellular components (24). It is induced under numerous cellular stresses, such as starvation, infection, protein aggregation and organelle damage $(25,26)$. Autophagy has complex functions in various stages of cancer and functions as a tumor suppressor that inhibits tumor initiation (27-29). In addition, autophagy reduces tumor growth and development by attenuating cellular metabolic stress, 
and thus acts as a survival pathway (23). Autophagosome construction is mediated by the autophagy-related 12 (ATG12)-ATG5-ATG16 system and LC3-I-phospholipid conjugate LC3-II, which is widely employed as an autophagy marker $(30,31)$.

Albeit the well-established anticancer effects of luteolin (17-19), its synergistic effects with TRAIL and the related molecular pathways are currently unclear. Therefore, in this study, we aimed to elucidate the molecular mechanisms underlying the outcome of luteolin and its synergistic effects when administered in combination with TRAIL in Huh7 liver cancer cells.

\section{Materials and methods}

Cells and cell culture. Human liver cancer cells (Huh7 and Hep3B) were obtained from the American Type Culture Collection (Global Bioresource Center, Manassas, VA, USA) and maintained in Dulbecco's modified Eagle's medium (Gibco BRL, Grand Island, NY, USA) containing 10\% fetal bovine serum (Sigma-Aldrich, St. Louis, MO, USA). For experimentation, the medium of the cells was changed to DMEM containing $1 \% \mathrm{FBS}$. Cells were cultured at $37^{\circ} \mathrm{C}$ with $5 \% \mathrm{CO}_{2}$ in humidified incubator.

Reagents. Luteolin (solvent, DMSO) was acquired from Cayman Chemical Co. (Ann Arbor, MI 48108, USA), TRAIL [solvent, phosphate-buffered saline (PBS)] was acquired from AbFrontier Co., Ltd. (Seoul, Korea). Chloroquine (CQ) diphosphate salt was purchased from Sigma-Aldrich (Merck $\mathrm{KGaA}$, Darmstadt, Germany) and CQ was dissolved in water to produce a $10 \mathrm{mM}$ stock solution. SP600125 $(10 \mu \mathrm{M})$ was acquired from the Beyotime Institute of Biotechnology (Shanghai, China). SP600125 and CQ added to the cells $1 \mathrm{~h}$ prior to treatment luteolin or TRAIL.

Cell viability assay. The liver cancer cells were plated in 12 -well plates and treated with luteolin $(0,5,10$ and $20 \mu \mathrm{M})$ for $18 \mathrm{~h}$ and exposed to TRAIL (200 ng/ml) for a further $2 \mathrm{~h}$. Cell morphology was assessed under a microscope (Nikon, Tokyo, Japan, magnification, x100), and cell viability was evaluated by the crystal violet staining solution kit from Sigma-Aldrich (Merck KGaA, Darmstadt, Germany) according to the manufacturer's instructions.

Trypan blue exclusion assay. Cell viability was evaluated by trypan blue exclusion assay (Sigma-Aldrich, St. Louis, MO, USA) using a hemocytometer (\#02-671-10; Thermo Fisher Scientific, Inc., Waltham, MA, USA). Following each treatment, cells were stained with $0.4 \%$ trypan blue for $5 \mathrm{~min}$ at room temperature. Unstained cells were regarded as viable, and stained cells were regarded as dead. The total cell number and the number of trypan blue-positive cells were counted using a light microscope in a blinded manner. The percentage of surviving cells was calculated using the formula: Number of stained cells/number of total cells x100. Each experiments was performed in triplicate.

Transmission electron microscopy (TEM) analysis. Following fixation of the cells in $2 \%$ glutaraldehyde (Electron Microscopy
Sciences, Hatfield, PA, USA) and 2\% paraformaldehyde (Electron Microscopy Sciences) in $0.05 \mathrm{M}$ sodium cacodylate (pH 7.2; Electron Microscopy Sciences) for $2 \mathrm{~h}$ at $4^{\circ} \mathrm{C}$ specimens were fixed in $1 \%$ osmium tetroxide (Electron Microscopy Sciences) for $1 \mathrm{~h}$ at $4^{\circ} \mathrm{C}$, dehydrated with increasing ethanol $(25,50,70,90$ and $100 \%)$ for 5 min each and embedded in epoxy resin (Embed 812; Electron Microscopy Sciences) for $48 \mathrm{~h}$ at $60^{\circ} \mathrm{C}$ according to the manufacturers' instructions. Ultrathin sections $(60 \mathrm{~nm})$ were prepared using an LKB-III ultratome (Leica Microsystems GmbH, Wetzlar, Germany) and were stained with $0.5 \%$ uranyl acetate (Electron Microscopy Sciences) for $20 \mathrm{~min}$ and $0.1 \%$ lead citrate (Electron Microscopy Sciences) for $7 \mathrm{~min}$ at room temperature. Images were recorded on a Hitachi $\mathrm{H} 7650$ electron microscope (Hitachi, Ltd., Tokyo, Japan; magnification, x10,000) installed at the Center for University-Wide Research Facilities (CURF) at Chonbuk National University.

Immunofluorescence staining. The Huh7 cells were cultured on poly-L-lysine-coated coverslips. Following differentiation and specific treatment, the cells were adjusted with $4 \%$ paraformaldehyde and permeablized with $0.1 \%$ Triton X-100. The cells were then incubated in a blocking solution followed by overnight incubation at $4^{\circ} \mathrm{C}$ with anti-p62 (cat. no. 5114; 1:1,000) and p-c-Jun N-terminal kinase (JNK, cat. no. 9255s) antibodies were from Cell Signaling Technology,Inc. (Danvers, MA, USA). After washing with PBS, the cells were incubated with secondary antibodies (Alexa Fluor ${ }^{\circledR}$ 488-conjugate; donkey polyclonal anti-rabbit, 1:500; Thermo Fisher Scientific, Inc.; cat. no. A-21206) and Texas-Red-X-conjugate (goat polyclonal anti-mouse, 1:500; Thermo Fisher Scientific, Inc.; cat. no. T-6390) for $2 \mathrm{~h}$ in the dark. In addition, 4',6'-diamidino-2phenyl-indole (DAPI; D9564; Sigma-Aldrich, St. Louis, MO, USA) was used to non-specifically stain the nuclei. Finally, immunostaining was visualized under a fluorescence microscope (\#451203, Nikon ECLIPSE 80i; Nikon Corporation, Tokyo, Japan; magnification, x400).

Western blot analysis. Western blot analysis was carried out as previously described (32). Briefly, RIPA lysis buffer was used to extract total proteins. The supernatant was collected by centrifuging at $13,282 \times \mathrm{g}$ at $4^{\circ} \mathrm{C}$ for $10 \mathrm{~min}$. Protein concentration was tested using the Pierce BCA Protein Assay kit (Thermo Fisher Scientific, Inc.). The samples $(30 \mu \mathrm{g})$ were separated on SDS-PAGE $(10 \%)$ and then blotted onto a polyvinylidene fluoride (PVDF) membrane (Merck Millipore, Bedford, MA, USA). The membrane was blocked with $5 \%$ non-fat dry milk at $25^{\circ} \mathrm{C}$ for $1 \mathrm{~h}$, and then incubated with primary antibodies overnight for $1 \mathrm{~h}$ at $4^{\circ} \mathrm{C}$. The $\beta$-actin antibody was from Sigma-Aldrich (cat. no. A2228; 1:2,000; Merck KGaA, Darmstadt, Germany), The antibodies against DR4 (cat. no. ab8414; 1:1,000) and DR5 (cat. no. ab181846; 1:1,000) were from Abcam (Cambridge, MA, USA); against LC3A/B (cat. no. 3868; 1:1,000), cleaved caspase-3 (cat. no. 9661; 1:500 ), p-JNK (cat. no. 9255s, 1:1,000), p62 (cat. no. 5114; 1:1,000), ATG5 (cat. no. 2630; 1:1,000) were from Cell Signaling Technology (Danvers, MA, USA) and the antibody against cleaved caspase-8 (cat. no. 551242; 1:1,000) was from BD Pharmingen/BD Biosciences (San Jose, CA, USA). The membrane was incubated with the corresponding horseradish 
A

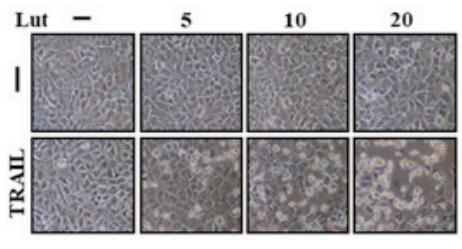

B

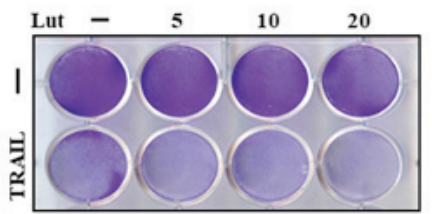

C

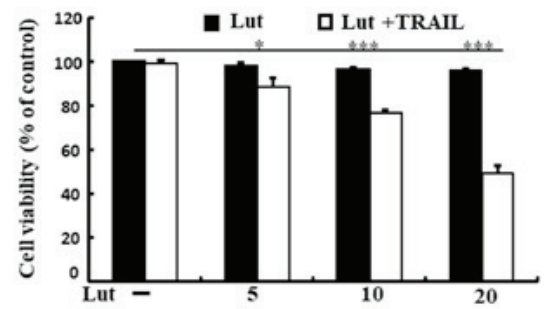

D

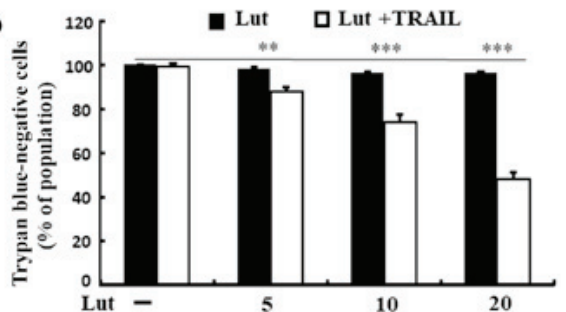

E Lut -

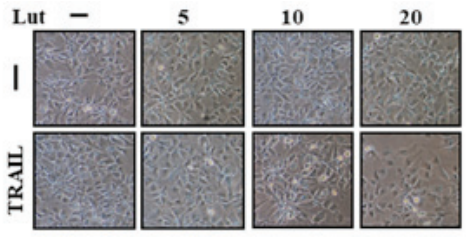

F

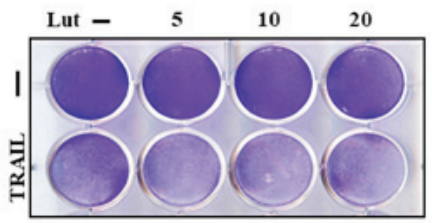

G

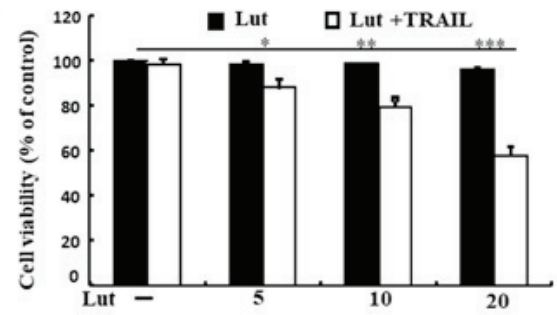

H

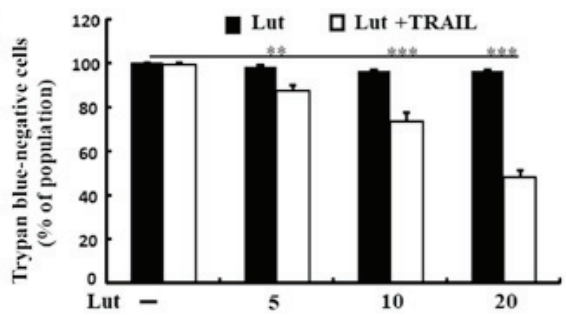

Figure 1. Luteolin sensitizes HCC cells to TRAIL-induced apoptosis. Huh7 and Hep3B cells were treated with luteolin $(0,5,10$ and $20 \mu \mathrm{M})$ for $18 \mathrm{~h}$ and then exposed to TRAIL (200 ng/ml) for a further $2 \mathrm{~h}$. (A and E) The morphology of the Huh7 and Hep3B cells was evaluated by interference light microscopy. (B and F) Cell viability was assessed by crystal violet staining of the Huh7 and Hep3B cells. (C and G) Bar diagram commencing the average density of crystal violet in the Huh7 and Hep3B cells. (D and H) Cell viability was evaluated with trypan blue dye exclusion assays in the Huh7 and Hep3B cells. "P<0.05, ${ }^{* *} \mathrm{P}<0.01$ and ${ }^{* * *} \mathrm{P}<0.001$, compared with the untreated group and treatment group. HCC, hepatocellular carcinoma; Lut, luteolin; TRAIL, tumor necrosis factor (TNF)-related apoptosis-inducing ligand.

peroxidase-conjugated secondary antibody (cat. no. 4410; Cell Signaling Technology; $1: 2,000$ ) at $25^{\circ} \mathrm{C}$ for $1 \mathrm{~h}$. The immunoreactive protein bands were visualized using an enhanced chemiluminescence detection system (GE Healthcare Life Sciences, Chalfont, UK).

Small interfering ATG5 RNA transfection. The Huh7 cells were seeded into 6 -well plates at a density of $1 \times 10^{4}$ cells/well. Following $48 \mathrm{~h}$ of incubation the cells reached $80 \%$ confluence and were transiently transfected with ATG5 siRNA using Lipofectamine ${ }^{\circledR} 2000$ (Invitrogen; Thermo Fisher Scientific, Inc.). The sequences for ATG5 siRNA (siRNA; oligo ID HSS114103; Invitrogen/Thermo Fisher Scientific) were as follows: Sense, 5'-GGCCUUUCAUUCAGAAGCUTT-3' and antisense, 5'-AGCUUCUGA AUGAAAGGCCTT-3'; the sequences for negative control (NC) were sense, 5'-UCUCCG AACGUGUCACGUTT-3' and anti-sense, 5'-ACGUGACAC GUUCGGAGAATT-3'. The oligodeoxynucleotides for the NC were obtained following scrambling of the siRNA oligodeoxynucleotide for ATG5, and were determined to not be associated with any mRNA sequence by BLAST. Cell transfection was performed, according to the protocol of the transfection kit manufacturer. Briefly, each sequence of MIF siRNA and $10 \mu \mathrm{l}$ Lipofectamine 2000 was diluted in serum-free medium $(250 \mu \mathrm{l})$ at room temperature for $5 \mathrm{~min}$, mixed together, and incubated for $30 \mathrm{~min}$ at room temperature. The mixture was subsequently administered to the Tca8113, SCC25 and HN5 cells, and after $5 \mathrm{~h}$ of incubation, the medium was replaced with complete medium.

Statistical analysis. Statistical analyses were carried out using GraphPad Prism software (version 5.03; GraphPad Software, Inc., La Jolla, CA, USA). All experiments were performed 3 times, and the data are expressed as the means \pm standard error. Significant differences between the control and treated samples were analyzed using one-way factorial analysis of variance (ANOVA), followed by Duncan's post-hoc test. A value of $\mathrm{P}<0.05$ was considered to indicate a statistically significant difference.

\section{Results}

Luteolin sensitizes HCC cells to TRAIL-induced apoptosis. Treatment with TRAIL or luteolin alone induced minimal or no cell death (Fig. 1). In addition, the treated cells exhibited no morphological differences from those of the control cells, revealing that the HCC cells were immensely resistant to TRAIL-mediated apoptosis. Co-treatment with TRAIL and increasing concentrations of luteolin markedly attenuated cell viability, increasing apoptotic bodies and decreasing 

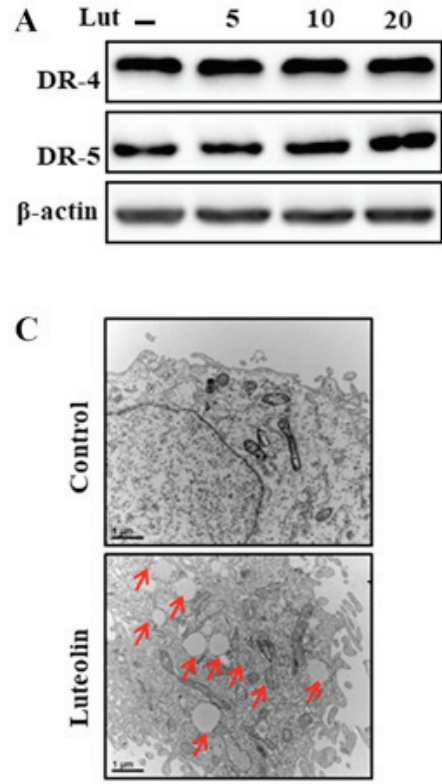

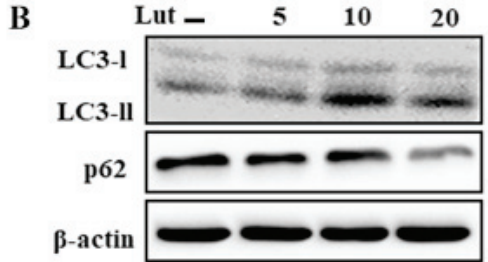

D

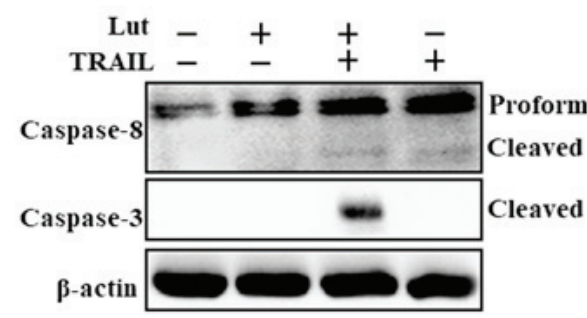

Figure 2. Luteolin induces autophagy and sensitizes cells to TRAIL-induced apoptosis. Huh7 cells were treated with luteolin $(20 \mu \mathrm{M})$ for $18 \mathrm{~h}$ and then to TRAIL for a further1 h. (A, B and D) DR4, DR5, LC3-II, p62, cleaved caspase-3 and cleaved caspase-8 were assessed by western blot analysis. (C) The formation of autophagosomes in the treated cells was observed by transmission electron microscopy. $\beta$-actin was detected as an input control. Lut, luteolin; TRAIL, tumor necrosis factor (TNF)-related apoptosis-inducing ligand; DR, death receptor.

attached cells compared to treatment with luteolin or TRAIL alone (Fig. 1A and E). Luteolin treatment sensitized both cells to TRAIL, but the sensitivity was higher in Huh7 cells than in Hep3B cells, and we focused on the Huh7 cells in this study. These findings suggested that luteolin effectively enhanced the sensitivity of human HCC cells to TRAIL-induced apoptosis.

Luteolin induces autophagy and sensitizes cells to TRAIL-induced apoptosis. As shown in Fig. 2A and B, luteolin treatment significantly upregulated LC3-II and downregulated p62 expression, which is consistent with DR5 upregulation. The results of TEM analysis revealed that numerous autophagic and vacant vacuoles were secreted in the luteolin-treated cells (Fig. 2C). The cells co-treated with luteolin and TRAIL also exhibited a higher expression of cleaved caspase-3 and cleaved caspase-8 (Fig. 2D). These outcomes suggested that luteolin can initiate autophagy in Huh7 cells.

Luteolin-mediates the augmentation of TRAIL-initiated apoptosis which is suppressed by the attenuation of autophagy. Co-treatment with luteolin, chloroquine and TRAIL suppressed cell death. Cell morphological analysis revealed that the apoptotic bodies were markedly reduced and the attaching cells were increased in the luteolin-, chloroquine- and TRAIL-treated cells. These results also confirmed that treatment with chloroquine attenuated the cell death induced by combined treatment with luteolin and TRAIL (Fig. 3A). Co-treatment with luteolin, TRAIL and chloroquine markedly increased the viability of the human HCC Huh7 cells (Fig. 3B-D). These outcomes suggested that chloroquine blocked TRAIL-induced and luteolin-mediated liver cancer cell death.

The inhibition of autophagy attenuates the enhancing effects of luteolin on the sensitivity of liver cancer cells to TRAIL-induced

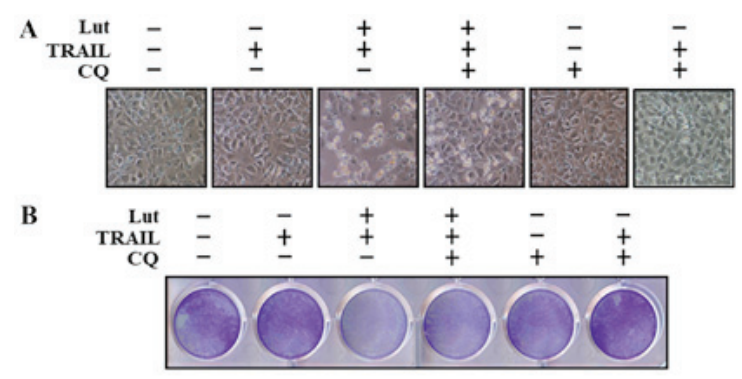

C

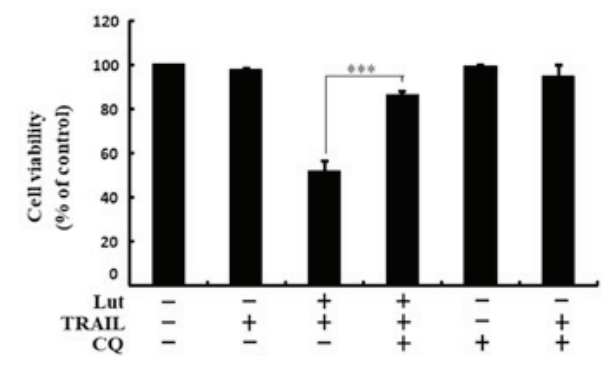

D

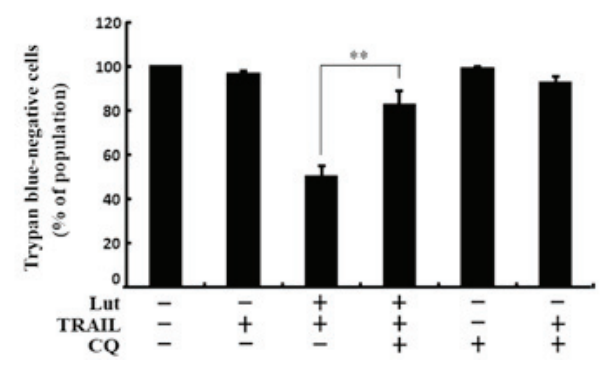

Figure 3. The luteolin-mediated augmentation of TRAIL-initiated apoptosis is suppressed by the attenuation of autophagy. Huh7 cells were treated with chloroquine prior to exposure to luteolin $(20 \mu \mathrm{M})$ for $18 \mathrm{~h}$ and then exposed to TRAIL for a further $2 \mathrm{~h}$. (A) The morphology of cells was evaluated by interference light microscopy. (B) Cellular viability was assessed by crystal violet staining. (C) Bar diagram commencing the average density of crystal violet. (D) Cellular viability was evaluated with trypan blue dye exclusion assays. ${ }^{* *} \mathrm{P}<0.01$ and ${ }^{* * *} \mathrm{P}<0.001$, compared with the untreated group and treatment group. Lut, luteolin; TRAIL, tumor necrosis factor (TNF)-related apoptosis-inducing ligand; $\mathrm{CQ}$, chloroquine. 

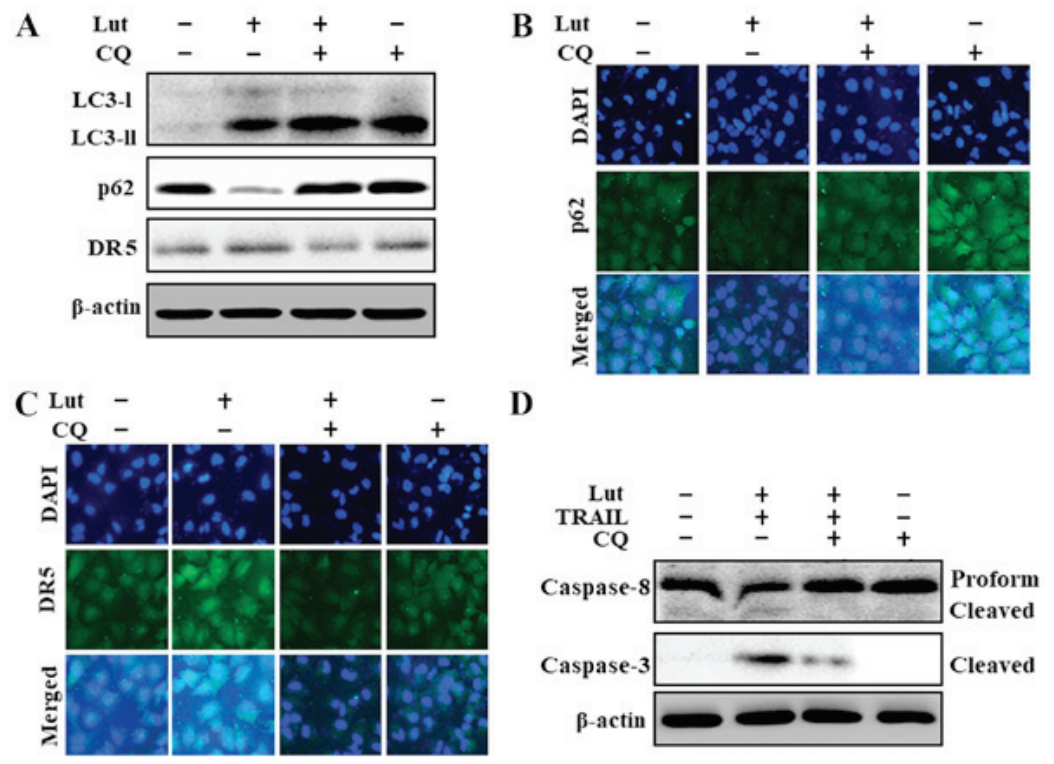

Figure 4. The inhibition of autophagy attenuates the enhancing effects of luteolin on TRAIL-induced apoptosis sensitization mediated by the promotion of the autophagic flux. Huh7 cells were treated with chloroquine prior to exposure to luteolin $(20 \mu \mathrm{M})$ for $18 \mathrm{~h}$ and then exposed to TRAIL for a further $1 \mathrm{~h}$. (A and D) DR5, LC3-II, p62, cleaved caspase-3 and cleaved caspase-8 were assessed by western blot analysis. (B and C) Cells were immunostained with p62, DR5 antibody (green) and evaluated for fluorescence. Lut, luteolin; TRAIL, tumor necrosis factor (TNF)-related apoptosis-inducing ligand; CQ, chloroquine.

apoptosis mediated by the promotion of the autophagic flux. The origination of autophagy was further confirmed based on the suppression of the autophagic flux following chloroquine treatment, which resulted in an increase in the accumulation of membrane-bound LC3-II, an increase in p62 levels, and the partial suppression of DR5 upregulation (Fig. 4A). The results of immunofluorescence staining also confirmed the increased p62 levels following treatment with chloroquine (Fig. 4B). Chloroquine also partially inhibited the upregulation DR5 which had been induced by luteolin and TRAIL (Fig. 4C). In addition, co-treatment with luteolin, TRAIL and chloroquine inhibited the increase in cleaved caspase- 8 and cleaved caspase-3 induced by luteolin and TRAIL (Fig. 4D). These outcomes suggested that the luteolin-induced augmentation of TRAIL-initiated apoptosis and the promotion of the autophagic flux were suppressed by chloroquine.

The enhancing effects of luteolin on the sensitivity of the cells to TRAIL-initiated apoptosis are suppressed by the genetic attenuation of autophagy. Co-treatment with luteolin, ATG5 siRNA and TRAIL prevented cell death. Cell morphological analysis revealed that the apoptotic bodies were markedly reduced and the attaching cells were increased in the luteolin-, ATG5 siRNA- and TRAIL-treated cells. The results confirmed that autophagy inhibition suppressed the cell death induced by treatment with luteolin and TRAIL, and also compared to transfection with the negative control (NC) (Fig. 5A). Co-treatment with luteolin and TRAIL, and transfection with ATG5 siRNA markedly increased the viability of the Huh7 HCC cells and significantly inhibited cell death (Fig. 5B-D). These outcomes suggested that autophagy inhibition blocked TRAIL- and luteolin-induced liver cancer cell death.

The genetic attenuation of autophagy suppresses luteolin-induced TRAIL-initiated apoptosis mediated by the activation of the autophagic flux. ATG5 knockdown

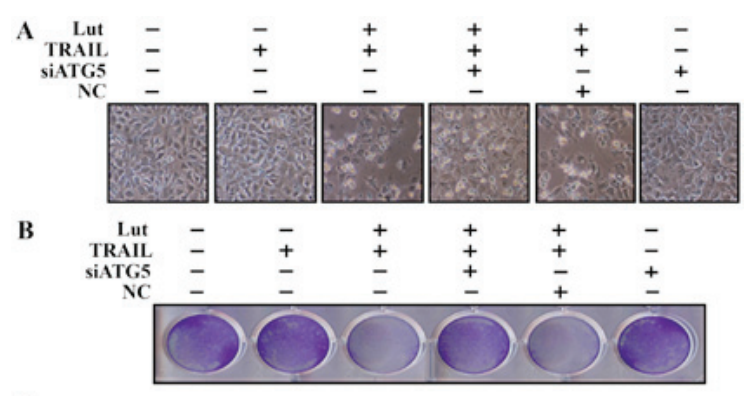

C

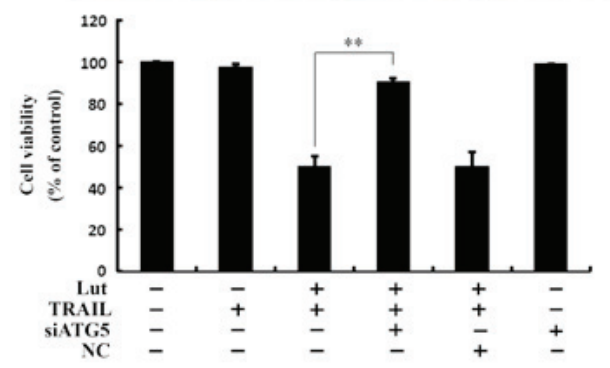

D

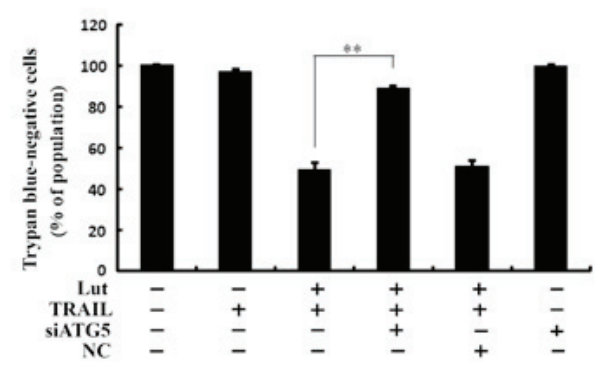

Figure 5. The luteolin-sensitized TRAIL-initiated apoptosis is suppressed by the genetic attenuation of autophagy. Huh7 cells were transfected with ATG5 siRNA or negative control siRNA for $24 \mathrm{~h}$ prior to exposure to luteolin $(20 \mu \mathrm{M})$ for $18 \mathrm{~h}$ and were then exposed to TRAIL for a further $2 \mathrm{~h}$. (A) The morphology of the cells was evaluated by interference light microscopy. (B) Cellular viability was assessed by crystal violet staining. (C) Bar diagram commencing the average density of crystal violet. (D) Cellular viability was evaluated by trypan blue dye exclusion assays. ${ }^{* *} \mathrm{P}<0.01$, compared with the untreated group and treatment group. Lut, luteolin; TRAIL, tumor necrosis factor (TNF)-related apoptosis-inducing ligand; siATG5, siRNA against autophagy-related 5; NC, negative control siRNA. 


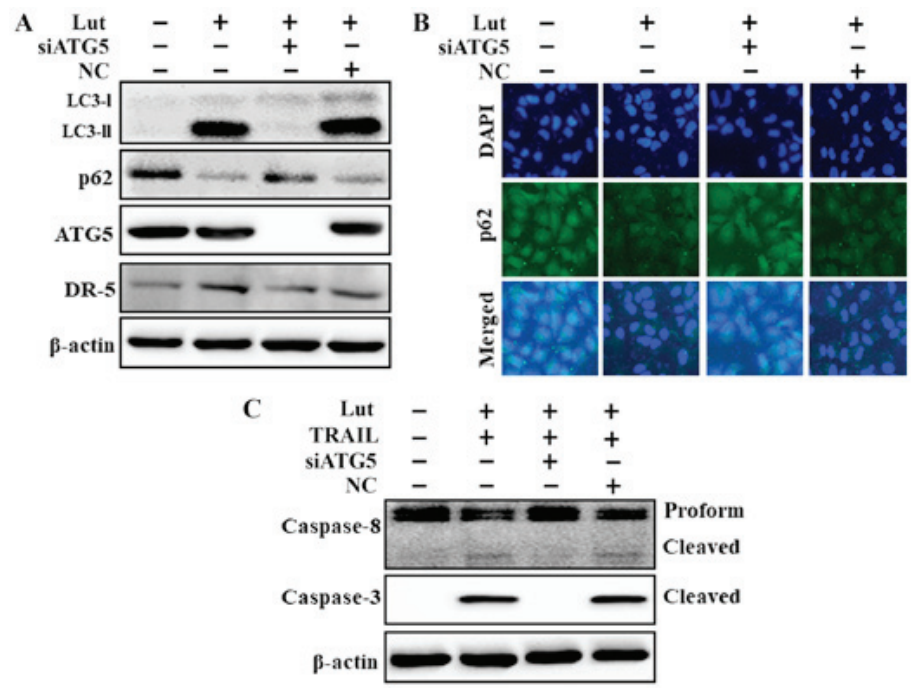

Figure 6. Genetic attenuation of autophagy suppresses the enhancing effects of luteolin on TRAIL-initiated apoptosis mediated through the activation of the autophagic flux. Huh7 cells were transfected with ATG5 siRNA or negative control siRNA for $24 \mathrm{~h}$ prior to exposure to luteolin (20 $\mu \mathrm{M})$ for $18 \mathrm{~h}$ and were then exposed to TRAIL for a further $1 \mathrm{~h}$. (A and C) DR5, LC3-II, p62, ATG5, cleaved caspase-3 and cleaved caspase-8 were assessed by western blot analysis. (B) Cells were immunostained with p62 antibody (green) and evaluated for fluorescence. Lut, luteolin; TRAIL, tumor necrosis factor (TNF)-related apoptosis-inducing ligand; siATG5, siRNA against autophagy-related 5; NC, negative control siRNA.

decreased the luteolin-induced expression of LC3-II and DR5 and markedly increased the p62 protein levels (Fig. 6A). The p62 protein levels determined based on immunofluorescence staining were consistent with the results of western blot analysis (Fig. 6B). However, co-treatment with luteolin, ATG5 siRNA and TRAIL attenuated the increase in Ac-cas3 and Ac-cas8 expression (Fig. 6C). These findings suggested that the luteolin-induced augmentation of TRAIL-initiated apoptosis mediated by the activation of the autophagic flux was suppressed by the genetic attenuation of autophagy.

Luteolin induces DR expression via JNK activation. Cell morphological analysis revealed that the apoptotic bodies were markedly reduced and the attaching cells were increased in JNK inhibitor (SP600125)-, luteolin- and TRAIL-treated cells (Fig. 7A). Co-treatment with luteolin, SP600125 and TRAIL reduced the cell apoptosis induced by luteolin and TRAIL (Fig. 7A and B). The results revelaed that luteolin increased JNK and decreased Akt phosphorylation (Fig. 7C). Pre-treatment with the JNK inhibitor (SP600125) significantly attenuated JNK phosphorylation and inhibited DR5 upregulation (Fig. 7D). The results of immunofluorescence staining also confirmed that SP600125 treatment significantly reduced JNK phosphorylation (Fig. 7E).

\section{Discussion}

TRAIL has been established as a significant potential cancer treatment due to its tumor specificity and safety (33). Untagged recombinant human TRAIL has been demonstrated to not induce any toxicity to normal human hepatocytes and is currently used as a clinical drug in the treatment of liver diseases (34). DRs, DR5 and DR4, are pro-apoptotic receptors of TRAIL and can interact with TRAIL to activate the extrinsic apoptotic signaling pathway (11). The antitumor effects of luteolin have been demonstrated in various tumor cells $(18,35-37)$. Luteolin can initiate cell cycle arrest
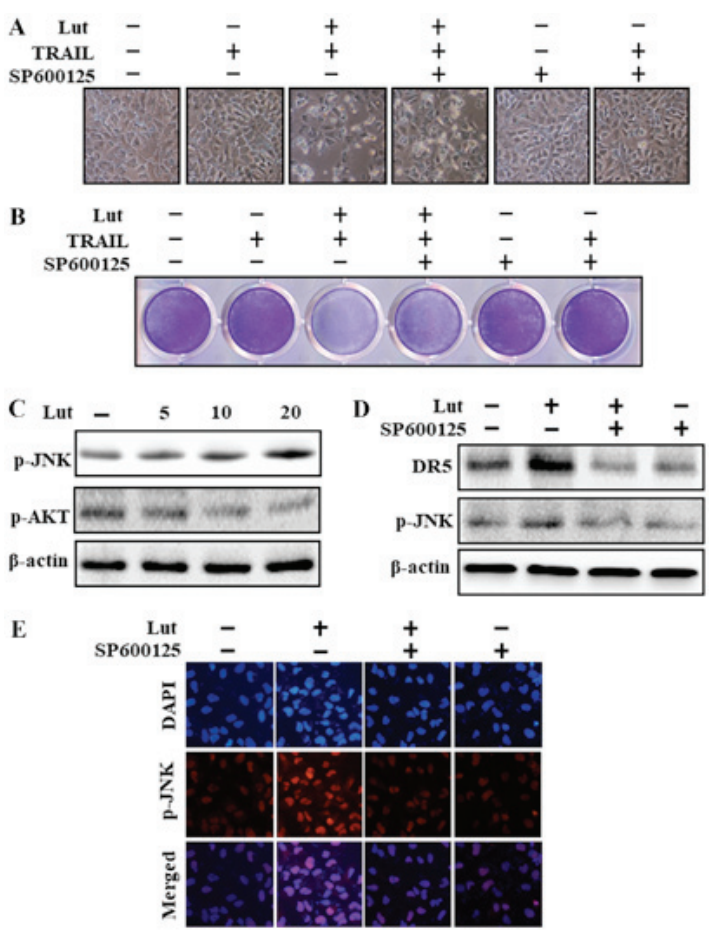

Figure 7. Luteolin induces DR expression via JNK activation. Huh7 cells were treated with SP600125 prior to exposure to luteolin $(20 \mu \mathrm{M})$ for $18 \mathrm{~h}$ and were then exposed to TRAIL for a further $2 \mathrm{~h}$. (A) The morphology of the cells was evaluated by interference light microscopy. (B) Cellular viability was assessed by crystal violet staining. (C and D) p-AKT, p-JNK and DR5 were assessed by western blot analysis. (E) Cells were immunostained with p-JNK antibody (red) and evaluated for fluorescence. Lut, luteolin; TRAIL, tumor necrosis factor (TNF)-related apoptosis-inducing ligand; DR, death receptor; JNK, the c-Jun N-terminal kinase.

and apoptosis, attenuate cell proliferation and metastatic advancement, and can inhibit doxorubicin-initiated cytotoxicity $(19,38,39)$. Autophagy is a lysosomal degradation system in which damaged proteins sustain cellular homeostasis $(25,40,41)$. Autophagy not only functions as a cell 
survival pathway, but also causes autophagic cell death when cells are subjected to stress (42-45).

Recent studies have demonstrated that HCC cells can develop resistance to TRIAL-initiated apoptosis $(46,47)$. In the current study, we demonstrated that treatment with TRAIL or luteolin alone initiated minimal or no apoptosis (Fig. 1). However, co-treatment with luteolin and TRAIL markedly initiates the death of Huh7 HCC cells, which are highly resistant to treatment with luteolin or TRAIL alone. This indication may lead to the development of a more effective and established mouse xenograft model and follow-up assessments using luteolin and perhaps, this may also treatment strategy may also be used in the future in the treatment of patients.

A recent study revealed that luteolin attenuated cancer cell proliferation and autophagy initiation (23). Furthermore, TRAIL-resistant tumor cells can be sensitized by chemotherapeutic agents that induce DR5 upregulation. Therefore, sensitization to TRAIL-induced apoptosis via DR5 upregulation is a promising approach for the treatment of TRAIL-resistant hepatoma cells (48-51). In the present study, the results from western blot analysis and immunocytochemistry indicated that luteolin treatment significantly increased the LC3-II levels and decreased the p62 levels in parallel with DR5 upregulation (Fig. 2). It has recently been demonstrated that autophagy is involved in drug-induced DR upregulation (52). The results of this study also suggested that chloroquine promoted cell survival and partially inhibited DR5 upregulation in Huh7 liver cancer cells (Figs. 3 and 4). However, for a reliable conclusion regarding DR5, additional studies are warranted with DR5 modulation, including DR5 knockout or knockdown experiments. In addition, the genetic attenuation of autophagy blocked the TRAIL-initiated and luteolin-mediated apoptosis of Huh7 cells (Figs. 5 and 6). Recent reports have also revealed that Akt, ERK and JNK can mediate DR upregulation (53-55). The findings of this study suggested that luteolin induced JNK activation via DR upregulation (Fig. 7).

In conclusion, the findings of this study demonstrate that JNK-mediated DR5 upregulation by luteolin sensitizes Huh7 liver cancer cells to TRAIL-induced cell death by promoting an autophagic flux. Combined treatment with luteolin and TRAIL may thus prove to be a useful and effective therapeutic regimen for some TRAIL-resistant cancers, including HCC.

\section{Acknowledgements}

Not applicable.

\section{Funding}

This study was supported by a grant from the National Research Foundation of Korea (NRF), funded by the Korean Government (2016R1A2B2009293).

\section{Availability of data and materials}

All data generated or analyzed during this study are included in this published article.

\section{Authors' contributions}

UMN and SYP designed the study. UMN performed the experiments. UMN and SYP analyzed the data and wrote the manuscript. Both authors reviewed the results and approved the final version of the manuscript.

\section{Ethics approval and consent to participate}

Not applicable.

\section{Patient consent for publication}

Not applicable.

\section{Competing interests}

The authors declare that they have no competing interests.

\section{References}

1. El-Serag HB and Rudolph KL: Hepatocellular carcinoma: Epidemiology and molecular carcinogenesis.Gastroenterology 132: 2557-2576, 2007

2. Sherman M: Epidemiology of hepatocellular carcinoma. Oncology 78 (Suppl 1): 7-10, 2010.

3. Balogh J, Victor D III, Asham EH, Burroughs SG, Boktour M, Saharia A, Li X, Ghobrial RM and Monsour HP Jr: Hepatocellular carcinoma: A review. J Hepatocell Carcinoma 3: 41-53, 2016.

4. Arii S: Molecularly targeted therapy for hepatocellular carcinoma from the basic and clinical aspects. Int J Clin Oncol 15: 234, 2010.

5. Llovet JM, Di Bisceglie AM, Bruix J, Kramer BS, Lencioni R, Zhu AX, Sherman M, Schwartz M, Lotze M, Talwalkar J, et al; Panel of Experts in HCC-Design Clinical Trials: Design and endpoints of clinical trials in hepatocellular carcinoma. J Natl Cancer Inst 100: 698-711, 2008.

6 . Wang S: The promise of cancer therapeutics targeting the TNF-related apoptosis-inducing ligand and TRAIL receptor pathway. Oncogene 27: 6207-6215, 2008.

7. Mahmood Z and Shukla Y: Death receptors: Targets for cancer therapy. Exp Cell Res 316: 887-899, 2010.

8. Allen JE and El-Deiry WS: Regulation of the human TRAIL gene. Cancer Biol Ther 13: 1143-1151, 2012.

9. Micheau O, Shirley S and Dufour F: Death receptors as targets in cancer. Br J Pharmacol 169: 1723-1744, 2013

10. Chen CY, Yiin SJ, Hsu JL, Wang WC, Lin SC and Chern CL: Isoobtusilactone A sensitizes human hepatoma Hep G2 cells to TRAIL-induced apoptosis via ROS and CHOP-mediated up-regulation of DR5. J Agric Food Chem 60: 3533-3539, 2012.

11. Pan G, O'Rourke K, Chinnaiyan AM, Gentz R, Ebner R, Ni J and Dixit VM: The receptor for the cytotoxic ligand TRAIL. Science 276: 111-113, 1997.

12. Walczak H, Degli-Esposti MA, Johnson RS, Smolak PJ, Waugh JY, Boiani N, Timour MS, Gerhart MJ, Schooley KA, Smith CA, et al: TRAIL-R2: A novel apoptosis-mediating receptor for TRAIL. EMBO J 16: 5386-5397, 1997.

13. Ashkenazi A, Pai RC, Fong S, Leung S, Lawrence DA, Marsters SA, Blackie C, Chang L, McMurtrey AE, Hebert A, et al: Safety and antitumor activity of recombinant soluble Apo2 ligand. J Clin Invest 104: 155-162, 1999.

14. Ashokkumar P and Sudhandiran G: Protective role of luteolin on the status of lipid peroxidation and antioxidant defense against azoxymethane-induced experimental colon carcinogenesis. Biomed Pharmacother 62: 590-597, 2008.

15. Nishitani Y, Yamamoto K, Yoshida M, Azuma T, Kanazawa K, Hashimoto $\mathrm{T}$ and Mizuno M: Intestinal anti-inflammatory activity of luteolin: Role of the aglycone in NF- $\mathrm{BB}$ inactivation in macrophages co-cultured with intestinal epithelial cells. Biofactors 39: 522-533, 2013.

16. Ashokkumar P and Sudhandiran G: Luteolin inhibits cell proliferation during Azoxymethane-induced experimental colon carcinogenesis via Wnt/ $\beta$-catenin pathway. Invest New Drugs 29: 273-284, 2011 
17. Huang X, Dai S, Dai J, Xiao Y, Bai Y, Chen B and Zhou M: Luteolin decreases invasiveness, deactivates STAT3 signaling, and reverses interleukin-6 induced epithelial-mesenchymal transition and matrix metalloproteinase secretion of pancreatic cancer cells. OncoTargets Ther 8: 2989-3001, 2015.

18. Han K, Meng W, Zhang JJ, Zhou Y, Wang YL, Su Y, Lin SC, Gan ZH, Sun YN and Min DL: Luteolin inhibited proliferation and induced apoptosis of prostate cancer cells through miR-301. OncoTargets Ther 9: 3085-3094, 2016.

19. Naso LG, Badiola I, Marquez Clavijo J, Valcarcel M, Salado C, Ferrer EG and Williams PAM: Inhibition of the metastatic progression of breast and colorectal cancer in vitro and in vivo in murine model by the oxidovanadium(IV) complex with luteolin. Bioorg Med Chem 24: 6004-6011, 2016.

20. Dia VP and Pangloli P: Epithelial-to-Mesenchymal Transition in Paclitaxel-Resistant Ovarian Cancer Cells Is Downregulated by Luteolin. J Cell Physiol 232: 391-401, 2017.

21. Youdim KA, Qaiser MZ, Begley DJ, Rice-Evans CA and Abbott NJ: Flavonoid permeability across an in situ model of the blood-brain barrier. Free Radic Biol Med 36: 592-604, 2004.

22. Jang S, Kelley KW and Johnson RW: Luteolin reduces IL-6 production in microglia by inhibiting JNK phosphorylation and activation of AP-1. Proc Natl Acad Sci USA 105: 7534-7539, 2008.

23. Cao Z, Zhang H, Cai X, Fang W, Chai D, Wen Y, Chen H, Chu F and Zhang Y: Luteolin promotes cell apoptosis by inducing autophagy in hepatocellular carcinoma. Cell Physiol Biochem 43: 1803-1812, 2017

24. Levine B and Klionsky DJ: Development by self-digestion: Molecular mechanisms and biological functions of autophagy. Dev Cell 6: 463-477, 2004

25. El-Khattouti A, Selimovic D, Haikel Y and Hassan M: Crosstalk between apoptosis and autophagy: Molecular mechanisms and therapeutic strategies in cancer. J Cell Death 6: 37-55, 2013.

26. Liang C: Negative regulation of autophagy. Cell Death Differ 17: $1807-1815,2010$

27. Giampietri C, Petrungaro S, Padula F, D'Alessio A, Marini ES, Facchiano A, Filippini A and Ziparo E: Autophagy modulators sensitize prostate epithelial cancer cell lines to TNF-alpha-dependent apoptosis. Apoptosis 17: 1210-1222, 2012

28. Lorenzi PL, Claerhout S, Mills GB and Weinstein JN: A curated census of autophagy-modulating proteins and small molecules: Candidate targets for cancer therapy. Autophagy 10: 1316-1326, 2014.

29. Yao D, Wang P, Zhang J, Fu L, Ouyang L and Wang J: Deconvoluting the relationships between autophagy and metastasis for potential cancer therapy. Apoptosis 21: 683-698, 2016.

30. Kabeya Y, Mizushima N, Ueno T, Yamamoto A, Kirisako T, Noda T, Kominami E, Ohsumi Y and Yoshimori T: LC3, a mammalian homologue of yeast Apg8p, is localized in autophagosome membranes after processing. EMBO J 19: 5720-5728, 2000.

31. Tanida I, Minematsu-Ikeguchi N, Ueno T and Kominami E: Lysosomal turnover, but not a cellular level, of endogenous LC3 is a marker for autophagy. Autophagy 1: 84-91, 2005.

32. Nazim UM, Jeong JK and Park SY: Ophiopogonin B sensitizes TRAIL-induced apoptosis through activation of autophagy flux and downregulates cellular FLICE-like inhibitory protein. Oncotarget 9: 4161-4172, 2017.

33. Kelley SK and Ashkenazi A: Targeting death receptors in cancer with Apo2L/TRAIL. Curr Opin Pharmacol 4: 333-339, 2004.

34. Volkmann X,Fischer U, Bahr MJ, Ott M, Lehner F, Macfarlane M, Cohen GM, Manns MP, Schulze-Osthoff $\mathrm{K}$ and Bantel $\mathrm{H}$ Increased hepatotoxicity of tumor necrosis factor-related apoptosis-inducing ligand in diseased human liver. Hepatology 46 : 1498-1508, 2007.

35. Yee SB, Choi HJ, Chung SW, Park DH, Sung B, Chung HY and Kim ND: Growth inhibition of luteolin on HepG2 cells is induced via 553 and Fas/Fas-ligand besides the TGF- $\beta$ pathway. Int J Oncol 47: 747-754, 2015.

36. Park SH, Ham S, Kwon TH, Kim MS, Lee DH, Kang JW, Oh SR and Yoon DY: Luteolin induces cell cycle arrest and apoptosis through extrinsic and intrinsic signaling pathways in MCF-7 breas cancer cells. J Environ Pathol Toxicol Oncol 33: 219-231, 2014

37. Sun DW, Zhang HD, Mao L, Mao CF, Chen W, Cui M, Ma R, Cao HX, Jing CW and Wang Z: Luteolin inhibits breast cancer development and progression in vitro and in vivo by suppressing notch signaling and regulating miRNAs. Cell Physiol Biochem 37: 1693-1711, 2015.
38. Sato Y, Sasaki N, Saito M, Endo N, Kugawa F and Ueno A Luteolin attenuates doxorubicin-induced cytotoxicity to MCF-7 human breast cancer cells. Biol Pharm Bull 38: 703-709, 2015.

39. Sui JQ, Xie KP and Xie MJ: Inhibitory effect of luteolin on the proliferation of human breast cancer cell lines induced by epidermal growth factor. Sheng Li Xue Bao 68: 27-34, 2016.

40. Mizushima N and Komatsu M: Autophagy: Renovation of cells and tissues. Cell 147: 728-741, 2011.

41. Quan W, Jung HS and Lee MS: Role of autophagy in the progression from obesity to diabetes and in the control of energy balance. Arch Pharm Res 36: 223-229, 2013.

42. Szczesny B, Brunyánszki A, Ahmad A, Oláh G, Porter C, Toliver-Kinsky T, Sidossis L, Herndon DN and Szabo C: Time-dependent and organ-specific changes in mitochondrial function, mitochondrial DNA integrity, oxidative stress and mononuclear cell infiltration in a mouse model of burn injury. PLoS One 10: $\mathrm{e} 0143730,2015$.

43. Li X, Xu HL, Liu YX, An N, Zhao S and Bao JK: Autophagy modulation as a target for anticancer drug discovery. Acta Pharmacol Sin 34: 612-624, 2013.

44. Lamy L, Ngo VN, Emre NC, Shaffer AL III, Yang Y, Tian E, Nair V, Kruhlak MJ, Zingone A, Landgren O, et al: Control of autophagic cell death by caspase-10 in multiple myeloma. Cancer Cell 23: 435-449, 2013.

45. Luo YH, Wu SB, Wei YH, Chen YC, Tsai MH, Ho CC, Lin SY, Yang CS and Lin P: Cadmium-based quantum dot induced autophagy formation for cell survival via oxidative stress. Chem Res Toxicol 26: 662-673, 2013.

46. Yamanaka T, Shiraki K, Sugimoto K, Ito T, Fujikawa K, Ito $M$, Takase $K$, Moriyama M, Nakano T and Suzuki A: Chemotherapeutic agents augment TRAIL-induced apoptosis in human hepatocellular carcinoma cell lines. Hepatology 32: 482-490, 2000.

47. Shankar S and Srivastava RK: Enhancement of therapeutic potential of TRAIL by cancer chemotherapy and irradiation: mechanisms and clinical implications. Drug Resist Updat 7: 139-156, 2004.

48. Chen JJ, Chou CW, Chang YF and Chen CC: Proteasome inhibitors enhance TRAIL-induced apoptosis through the intronic regulation of DR5: involvement of NF-kappa B and reactive oxygen species-mediated p53 activation. J Immunol 180: 8030-8039, 2008.

49. Kim H, Kim EH, Eom YW, Kim WH, Kwon TK, Lee SJ and Choi KS: Sulforaphane sensitizes tumor necrosis factor-related apoptosis-inducing ligand (TRAIL)-resistant hepatoma cells to TRAIL-induced apoptosis through reactive oxygen species-mediated up-regulation of DR5. Cancer Res 66: $1740-1750,2006$

50. Jung EM, Lim JH, Lee TJ, Park JW, Choi KS and Kwon TK: Curcumin sensitizes tumor necrosis factor-related apoptosis-inducing ligand (TRAIL)-induced apoptosis through reactive oxygen species-mediated upregulation of death receptor 5 (DR5). Carcinogenesis 26: 1905-1913, 2005.

51. Horinaka M, Yoshida T, Shiraishi T, Nakata S, Wakada M and Sakai T: The dietary flavonoid apigenin sensitizes malignant tumor cells to tumor necrosis factor-related apoptosis-inducing ligand. Mol Cancer Ther 5: 945-951, 2006.

52. Chen L, Meng Y, Guo X, Sheng X, Tai G, Zhang F, Cheng H and Zhou Y: Gefitinib enhances human colon cancer cells to TRAIL-induced apoptosis of via autophagy- and JNK-mediated death receptors upregulation. Apoptosis 21: 1291-1301, 2016.

53. Cheng H, Hong B, Zhou L, Allen JE, Tai G, Humphreys R, Dicker DT, Liu YY and El-Deiry WS: Mitomycin C potentiates TRAIL-induced apoptosis through p53-independent upregulation of death receptors: Evidence for the role of c-Jun N-terminal kinase activation. Cell Cycle 11: 3312-3323, 2012.

54. Thamkachy R, Kumar R, Rajasekharan KN and Sengupta S: ERK mediated upregulation of death receptor 5 overcomes the lack of p53 functionality in the diaminothiazole DAT1 induced apoptosis in colon cancer models: Efficiency of DAT1 in Ras-Raf mutated cells. Mol Cancer 15: 22, 2016.

55. Shoeb M, Ramana KV and Srivastava SK: Aldose reductase inhibition enhances TRAIL-induced human colon cancer cell apoptosis through AKT/FOXO3a-dependent upregulation of death receptors. Free Radic Biol Med 63: 280-290, 2013. 